\title{
Monitoring of the process of safe divergence on the part of an unmanned vessel
}

\author{
Sergey Smolentsev ${ }^{1, *}$ and Anatoly Sazonov ${ }^{1}$ \\ ${ }^{1}$ Admiral Makarov State University of Maritime and Inland Shipping, Russia
}

\begin{abstract}
The paper deals with the problem of control of maneuvers in the divergence of vessels. In a situation of dangerous convergence of vessels, one of them must take a maneuver for safe divergence in accordance with the COLREGS (International Rules of Preventing Collision at Sea). In this case, the vessel, which is given way, must maintain its course and speed. However, it must also monitor compliance with the divergence obligations by the oncoming vessel. The problems arising in the case when the vessel, which is given way, is an unmanned vessel controlled by an automatic agent. The reasons are given for the fact that the behavior of the oncoming vessel may differ from what is expected from the agent managing the vessel to which they must give way. For these cases, the criteria are provided by which the agent operating the unmanned vessel can detect the situation of non-compliance by another vessel with its divergence obligations, in order to take timely action to avoid a collision by maneuvering agent's vessel in accordance with the COLREGS.
\end{abstract}

\section{Introduction}

A lot of attention has recently been paid to the creation of unmanned vessels. To implement the project of an unmanned vessel, it is necessary to solve a whole range of problems. In particular, the problem of automatic divergence from other vessels.

A number of works are devoted to solving the main problems of automatic divergence, namely, the problem of assessing the situation and developing a solution for safe divergence [1-4].

Currently, active research is being conducted in the field of developing systems for safe divergence of unmanned vessels. The work [5] provides an overview of current research in this area. In particular, the work [6] discusses the use of neural networks in algorithms for safe divergence, the works $[7,8]$ discusses the use of the VO (Velocity obstacle) algorithm, and in the work [9] - the OZT method.

In the works of the author $[10,11]$, the issues of solving the problem of safe divergence and cooperative maneuvering of unmanned vessels controlled by automatic agents are also considered.

However, the issue of monitoring the implementation of the found solution for the divergence by automatic agents operating different vessels remains open.

\footnotetext{
${ }^{*}$ Corresponding author: smolencevsv@gumrf.ru
} 
This paper deals with the problem of controlling the actions of the vessels to implement the decision on the divergence.

\section{Methods and Materials}

In case of detection of a situation of dangerous approach to another vessel, the collision prevention system on an unmanned vessel solves the problem of ensuring a safe divergence. The solution to this problem will be a new trajectory of the vessel, which is obliged to give way in this situation. This trajectory is chosen in such a way as to ensure a safe divergence not only with the dangerous vessel, but also with all other vessels in the given water area.

For the sake of simplicity, we will consider a meeting of two vessels, although in the situation of a meeting of several vessels, the problem can only get worse. Let us denote: A the considered unmanned vessel, which is controlled by an automatic agent, B - an oncoming vessel, controlled by an agent, which can be either automatic (also an unmanned vessel) or by a skipper.

If in the current situation of a dangerous approach, the vessel A should give way, then it takes the maneuvers calculated by it to safely divergence from the vessel B. However, if the vessel giving way is an oncoming vessel (B), then an uncertain situation may arise. It consists in the fact that the agent who manages the unmanned vessel $\mathrm{A}$ is not sure that the decision on the divergence received by agent will be implemented by the oncoming vessel B. This is exactly the situation that will be considered here.

The problem is that the maneuver for safe divergence is calculated by the agent operating the unmanned vessel $\mathrm{A}$, and the divergence maneuver itself must be performed by the vessel B (obliged in this case according to the IPPCC to give way). However, the oncoming vessel B may not behave as expected by the agent operating the unmanned vessel A, for various reasons, among which are the following:

1. Agent operating the vessel B, may assess the situation of rapprochement or otherwise define it as dangerous because:

1.1. Agent uses other hazard criteria (shape and size of the domains of safety for agent's and others vessels, using the same security domains for all vessels, etc.);

1.2. The planned route of the vessel B is different from the route, forecasted in the system of divergence on the vessel A and in fact, it is not dangerous.

2. The maneuver obtained in the divergence system used on vessel A cannot be implemented by vessel $\mathrm{B}$, because:

2.1. The parameters of the model of vessel $B$ used to calculate the maneuver in the divergence system on vessel A differ from the dynamic characteristics of the real vessel B;

2.2. External factors that are not taken into account in the divergence system on the vessel A when making recommendations (for example, hydro-meteorological conditions, depths, etc.) prevent the implementation of the calculated maneuver.

3. The agent operating vessel $B$ plans to perform different maneuver than the maneuver calculated by the divergence system on vessel A, since when choosing the maneuver:

3.1. On the vessel B the COLREGS are treated differently in this particular situation of rapprochement;

3.2. The safe divergence systems on the vessels A and B are configured differently and, accordingly, issue different divergence decisions;

3.3. Vessels $\mathrm{A}$ and $\mathrm{B}$ take into account a different set of external conditions (for example, when receiving a decision, vessel A or B does not take into account the presence of other vessels, depth, visibility conditions or other restrictions on decisions in these conditions). 
In case of a meeting of two unmanned vessels, the agents who manage these vessels should be able to contact each other to develop a common understanding of the current situation of rapprochement, an unambiguous assessment of the degree of its danger and to develop an agreed solution for the divergence. For this purpose, it is important to implement the use of the context of the situation proposed in work [12] in the automatic divergence systems on these vessels, as well as to implement the protocol of information exchange between agents described in work [13].

In case of a meeting of an unmanned vessel and a vessel operated by a skipper, the problem of uncertainty in the actions of the oncoming vessel becomes even more acute, since the agent operating the unmanned vessel A will not be able to organize an effective exchange of information with the agent operating the vessel B (skipper).

In any case, it is important that the agent operating the vessel knows exactly how the oncoming vessel is being operated (by the crew, remotely or autonomously). This information is proposed to be transmitted as mandatory in the AIS system.

\section{Results}

As mentioned in the previous section, when vessels diverge, there is uncertainty in the behavior of the oncoming vessel. And it increases if it is not possible to organize an effective exchange of information between the agents operating the vessels, as a result of which they could agree on the implementation of a coordinated maneuver for a safe divergence.

However, in any case, even if the maneuver is agreed upon, the agent managing the vessel to which the way must be given is obliged to monitor the implementation of the agreements reached. And even more so if such agreements could not be reached. After all, if the vessel that is supposed to give way does not start the divergence maneuver in a timely manner, the vessel that is supposed to get way can (and then must), according to Rule 17 of the COLREGS, independently take measures for safe divergence.

Thus, after receiving (and better agreement with the agent of the oncoming vessel) a divergence maneuver, the agent of the vessel that is supposed to get way is obliged to monitor the behavior of the oncoming vessel and monitor its performance of this maneuver. It is important to detect the situation of non-maneuvering by an oncoming vessel in a timely manner, in order to assess the situation in a timely manner and recalculate the decision on maneuvering. Perhaps already your own ship.

In the case of an agreed maneuver when two unmanned vessels meet and implement a protocol of their interaction to detect the situation of the execution or non-execution of the agreed maneuver by the oncoming vessel $\mathrm{B}$, the agent of vessel $\mathrm{A}$ has the following information (figure 1):

1. The initial trajectory, which is constructed by the forecast subsystem (predictor) on the vessel A using the original route of the vessel B and its actual movement before maneuvering.

2. The trajectory of the maneuver of the vessel $B$, which is based on the recommended route for safe divergence, agreed by agents $\mathrm{A}$ and $\mathrm{B}$.

3. The trajectory of the actual movement of the vessel $\mathrm{B}$, which is built according to AIS and (or) radar data. 


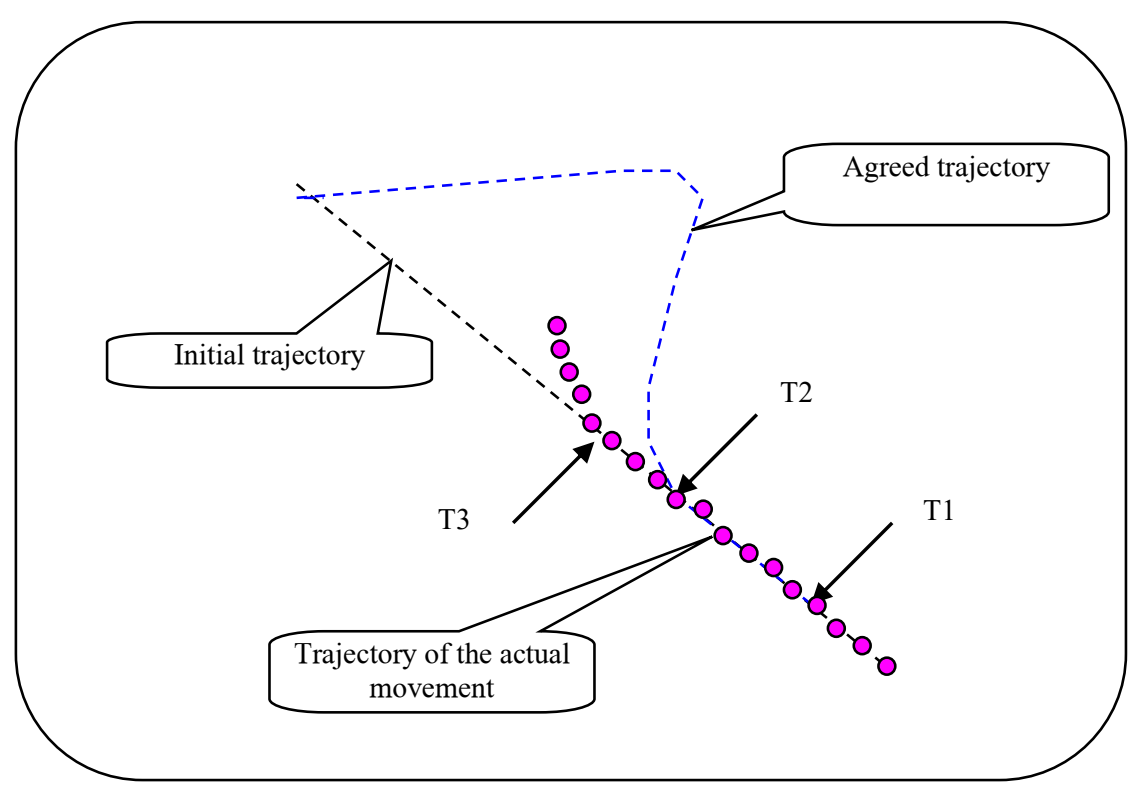

Fig. 1. Oncoming vessel B, the agent of vessel A.

Figure 1 shows:

T1 - the moment when the agents of the unmanned vessels reach an agreement on the maneuver of the vessel B;

T2 - the moment of the expected start of the transition of the vessel B to a new trajectory in accordance with the agreed divergence maneuver;

T3 - the moment of real deviation from the previous trajectory in accordance with the maneuver actually taken by the vessel B for divergence. Ideally, it should be: $\mathrm{T} 2=\mathrm{T} 3$.

The criterion for non-compliance with the agreed maneuver is the deviation of the trajectory of the actual movement of the vessel B from the trajectory determined as a result of the agreements reached. In fact, this is the absence of a change in the trajectory parameters at the moment T2. However, it should be borne in mind that:

1. The position of the vessel $\mathrm{B}$ is determined with an error.

2. The trajectory of movement of the vessel B, even fulfilling the agreements reached, cannot exactly coincide with the calculated trajectory due to the discrepancy between the model of the vessel B used in the calculations of the maneuver and the real dynamic characteristics of the vessel, as well as incomplete consideration of the external sailing conditions. 


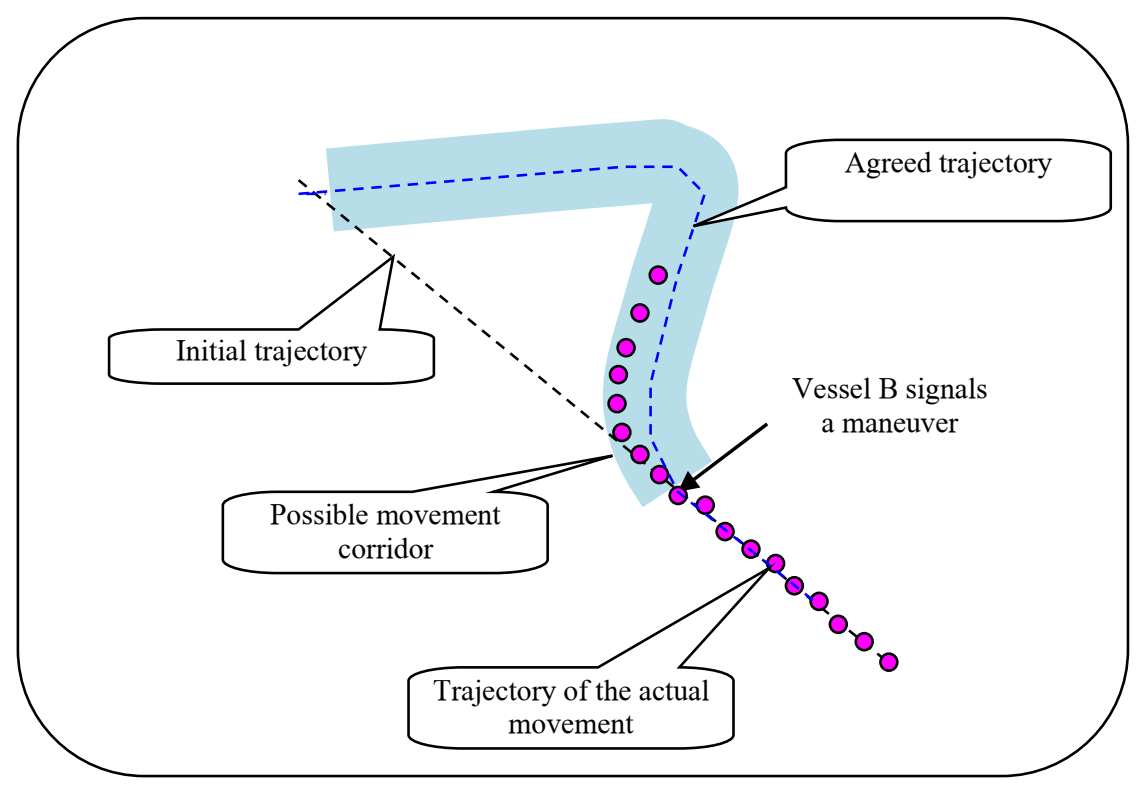

Fig. 2. False triggering of the detector of violation of agreements by agent of vessel A.

Thus, a false triggering of the detector of violation of agreements by agent of vessel A. To solve this problem, we can use (figure 2):

1. Mandatory notification by the agent operating the vessel B about the beginning of the execution of the agreed maneuver.

2. Setting the value of the possible deviation of the real trajectory of the vessel B from its actual trajectory, taking into account the above errors. Thus, a certain "corridor" is set around the calculated maneuver trajectory, the vessel B movement within which does not lead to a false trigger of the non-compliance detector.

In the case of meeting of an unmanned vessel $\mathrm{A}$ and a skipper-controlled vessel $\mathrm{B}$, the problem under consideration becomes more acute. In this case, the agent operating ship A cannot agree with the agent operating ship B on an agreed maneuver in automatic mode. The agent operating vessel A cannot even know for certain that the agent operating ship B (the skipper) is monitoring the situation, assessing it as dangerous, and planning to perform any maneuvers for a safe divergence. In this case, the decisions of Agent of vessel A should be based on the following assumptions about decisions of Agent operating vessel B:

- the decision on the degree of danger of the situation coincides with the decision of Agent of vessel A;

- maneuvering solutions comply with the COLREGS.

The automatic divergence system installed on vessel A can provide the agent of that vessel with a maneuvering solution that ensures safe divergence. But if this decision concerns the maneuvering of vessel $\mathrm{B}$, then it is not necessary to expect that the skipper who manages vessel $\mathrm{B}$ will make the same decision. In addition, at the start of the maneuver, the skipper of the vessel B will not notify the agent of the vessel A about this, which certainly complicates the task of tracking the execution of this maneuver.

In this case, it is proposed to use the following approach to solving the problem of detecting the failure of the divergence maneuver (figure 3).

1. The worst maneuver that can be performed by vessel B to safely divergence from vessel $\mathrm{A}$ is calculated. This is usually the latest maneuver, when it is still possible to ensure a safe divergence by the actions of only vessel B. 
2. The sector of possible maneuvers is constructed, limited by the found worst maneuver.

3. The position of the vessel $\mathrm{B}$ is monitored and if it continues to move along the previous trajectory and goes beyond the sector of possible maneuvers, the situation of failure to perform the divergence maneuver is detected and a decision is made on the need for divergence by the maneuver of the vessel $\mathrm{A}$.

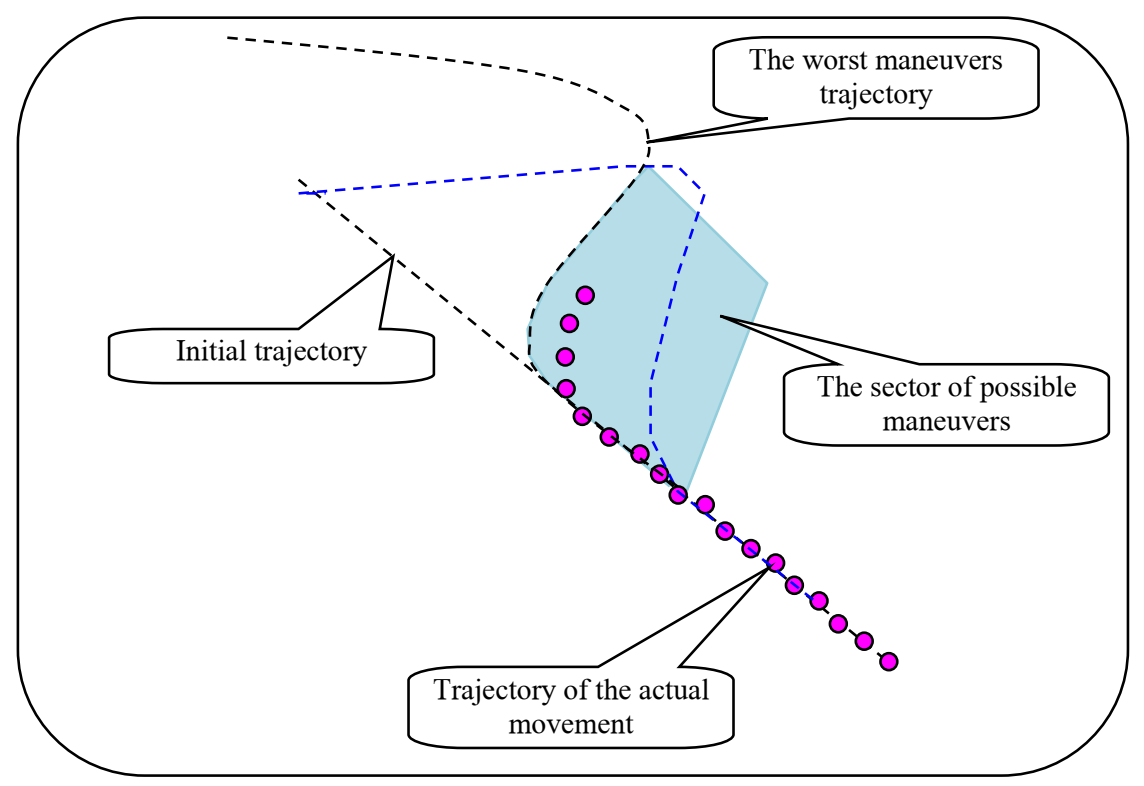

Fig. 3. Solving the problem of detecting the failure of the divergence maneuver.

\section{Discussion}

If, using the methods described in the previous section, the exit of the oncoming vessel B is detected outside the permissible maneuver area, the agent of vessel A makes a decision on the failure of vessel B to fulfill its obligations for divergence. And then he will be forced to calculate such a maneuver, so that the actions of only his vessel avoid a collision. Therefore, when assigning this permissible area, both in the case of a corridor of possible movement for an unmanned vessel, and in the case of a sector of possible maneuvers for a vessel controlled by a skipper, it is necessary to provide a reserve of time and free water space for performing its own maneuver.

In addition, when performing this forced maneuver, the agent of vessel A must take all possible actions to notify the agent operating the oncoming vessel $\mathrm{B}$ of his intentions to perform the maneuver. In the case of unmanned vessels, this can be done by means of a protocol for the exchange of information between automatic agents. In any case, the unmanned vessel must notify the intention to maneuver by other means, such as by means of sound and light signals prescribed by Rule 34 of the COLREGS. In this way, it will be able to warn the skipper operating the vessel B and the skippers (agents) operating other vessels in the water area about its intentions. 


\section{Conclusion}

The paper considers the problem of controlling the maneuvers of an oncoming vessel when vessels diverge at sea. In the case of a meeting of unmanned vessels, it is necessary to monitor the implementation of the maneuver agreements reached in the process of cooperative solution of the problem of divergence. In the case of a meeting of unmanned vessel and a vessel controlled by a skipper, the uncertainty in the maneuver performed by him increases significantly.

The approaches proposed in this paper allow solving the problem of detecting the situation of failure to perform a maneuver by a vessel that is obliged to give way in accordance with the COLREGS. This will allow making a timely decision on maneuvering an unmanned vessel, which will avoid a collision.

In this paper, the course maneuvers are considered as divergence maneuvers. Speed maneuvers require additional research.

\section{References}

1. S.J. Li, J.L. Liu, R.R. Negenborn, Ocean Eng. 181, 212-226 (2019) https://doi.org/10.1016/j.oceaneng.2019.03.054

2. D. Kim, K. Hirayama, T. Okimoto, J. Navig. 70(4), 699-718 (2017) https://doi.org/10.1017/s037346331700008x

3. D.G. Kim, K. Hirayama, G.K. Park, J. Adv. Comput. Intellig. Intellig. Inform. 18(5), 839-848 (2014) https://doi.org/10.20965/jaciii.2014.p0839

4. Y.X. He, Y. Jin, L.W. Huang, Y. Xiong, P.F. Chen, J.M. Mou, Ocean Eng. 140, 281291 (2017) https://doi.org/10.1016/j.oceaneng.2017.05.029

5. Y. Huang, L. Chen, P. Chen, R.R. Negenborn, P.H.A.J.M. van Gelder, Safety Science 121, 451-473 (2020) https://doi.org/10.1016/j.ssci.2019.09.018

6. T. Praczyk, Neurocomputing 149, 559-572 https://doi.org/10.1016/j.neucom.2014.08.018

7. Y. Huang, P.H.A.J.M. van Gelder, Y. Wen, Ocean Eng. 151, 308-321 (2018) https://doi.org/10.1016/j.oceaneng.2018.01.001

8. Y. Huang, L. Chen, P.H.A.J.M. van Gelder, Ocean Eng. 173, 142-156 (2019) https://doi.org/10.1016/j.oceaneng.2018.12.053

9. J. Fukuto, H. Imazu, IFAC Proc. 46(33), 91-96 https://doi.org/10.3182/20130918-4-jp-3022.00044

10. S.V. Smolentsev, Vestnik Gosudarstvennogo universiteta morskogo i rechnogo flota imeni admirala S.O. Makarova 2(36), 7-16 (2016) https://doi.org/10.21821/2309-51802016-8-2-7-16

11. S.V. Smolentsev, A.E. Sazonov, and Y.M. Iskanderov, Vestnik Gosudarstvennogo universiteta morskogo i rechnogo flota imeni admirala S. O. Makarova 10.4, 687-695 (2018) https://doi.org/10.21821/2309-5180-2018-10-4-687-695

12. S.V. Smolentsev, A.E. Sazonov, A.E. Pelevin, Vestnik Gosudarstvennogo universiteta morskogo i rechnogo flota imeni admirala S. O. Makarova 12.2, 221-229 (2020) DOI: 10.21821/2309-5180-2020-12-2-221-229

13. S. Smolentsev, International Scientific Conference Energy Management of Municipal Facilities and Sustainable Energy Technologies EMMFT 2019. Advances in Intelligent Systems and Computing Springer, Cham 1258, 442-452 (2019) https://doi.org/10.1007/978-3-030-57450-5_38 